\title{
Interference Cancellation Techniques in Heterogeneous Networks
}

\author{
Sarah Imam, Ahmed El-Mahdy*
}

\begin{abstract}
The fifth generation (5G) cellular wireless networks are heterogeneous networks that will provide higher data rates, enhanced quality-of-experience (QoE) and reduced latency. Interference is one of the main problems in such systems. In this paper, a proposed cross-tier uplink interference alignment algorithm for coexisting two-tier networks is introduced. First at each receiver, the sum of the square of the channel gains from each macro user to this receiver is calculated and the average of these values is taken as a threshold. Macro users whose sum values are greater than this threshold are selected and aligned at the femto receiver. This alignment is performed by using precoders at the transmitters. Then, Zero Forcing technique is applied at the receivers in order to null the aligned interference signals. Numerical results demonstrate the superior performance of the proposed algorithm.
\end{abstract}

Keywords: Wireless communications, heterogeneous networks, interference alignment, Zero Forcing.

\section{Introduction}

The 5G networks will consist of nodes/cells with heterogeneous characteristics and capacities (e.g., macrocells, small cells [such as femtocells and picocells], Device to Device (D2D) user equipment [UE] which is based on the direct communication between two mobile users without traversing the Base Station (BS) or the core network), which will result in a multi-tier architecture. Due to increasing complexity in network management and coordination among multiple network tiers, the network nodes will have the capability of self-organization (e.g., autonomous load balancing, interference minimization, spectrum allocation, power adaptation etc.) [16]. Also, a UE can have simultaneous active connections to more than one base station (BS) or access point (AP) using the same or different radio access technologies (RATs). The heterogeneous nodes (e.g., UEs, BSs, smart machines, wearable devices etc.) can be integrated through a unified (possibly cloud-based) network to provide seamless connectivity. The communication efficiency in 5G systems will be improved by incorporating techniques such as coordinated multipoint (CoMP) joint

* German University in Cairo, New Cairo City, Main Entrance El Tagmoa El Khames, Cairo, Egypt. \{sarah.mohamed-imam, ahmed.elmahdy\}@guc.edu.eg 
transmission and reception, network-assisted interference cancellation and suppression, spectrum reuse (e.g., non-orthogonal multiple access), and three-dimensional or fulldimensional MIMO [1]. In addition, the use of a large number of remote radio heads (RRHs) connected to central processing nodes (e.g., clouds) with the high-speed backhaul/fronthaul is also envisioned [14].

As shown in Figure 1-1, the 5G cellular will be a mulit-tier heterogeneous network consisting of macrocells along with a large number of low power nodes such as (small cells, relays, remote radio heads [RRHs]) along with the provisioning for Peer to Peer (P2P) where interconnected nodes ("peers") share resources with each other without the use of a centralized administrative system (such as D2D and Machine to Machine (M2M) in which devices communicate directly using any communication channel) communication. The deployments of heterogeneous nodes in 5G systems will significantly have much higher density than today's conventional single-tier (e.g., macrocell) networks [1]. The heterogeneity of different classes of BSs (e.g., macrocells and small cells) provides flexible coverage area and improves spectral efficiency. By reducing the size of the cell, the area spectral efficiency is increased through higher spectrum reuse. Additionally, the coverage can be improved by deploying small cells indoors (such as home, office buildings, public vehicles etc.). Wireless P2P communication (e.g., D2D/M2M communication among UEs and autonomous sensors/actuators) underlaying cellular architecture can significantly increase the overall spectrum and energy efficiency of the network. In addition, the network-controlled P2P communications in 5G systems will allow other nodes (such as relay or M2M gateway), rather than the macrocell $\mathrm{BS}$, to control the communications among P2P nodes. Given that the inter-tier and intra-tier interferences are well managed, the adoption of multiple tiers in the cellular network architecture will provide better performance in terms of coverage, capacity, spectral efficiency, and power consumption [8].

The co-operation of heterogeneous networks with different characteristics and capacities results in a multi-tier architecture that has the advantages of providing higher data rates, reduced latency and enhanced quality of experience but, this multi-tier architecture causes an interference problem. Interference occurs when a certain receiver receives signals from un-intended transmitters and this causes degradation in the system performance. Hence, in order to maintain a high system performance with a high Quality of Service (QoS), the interference problem should be solved. Interference Alignment (IA) is one of the solutions, by applying IA, the interference signals at the undesired receiver are aligned in the same sub-space. Interference suppression techniques are then applied to suppress the aligned interference signals. 


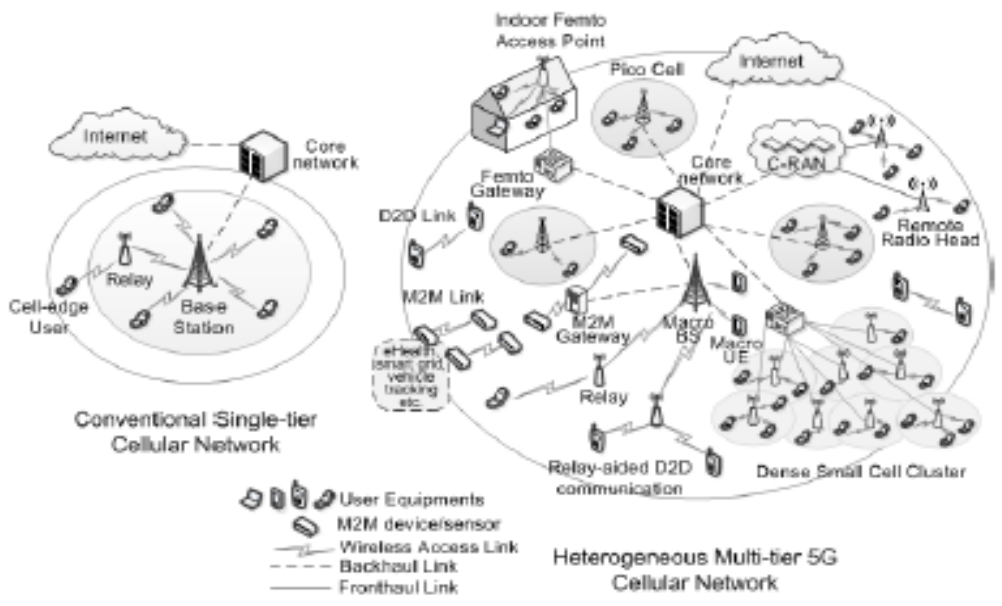

Figure 1-1 The aspects of 5G networks and devices [1]

In order to reduce the load on the wireless cellular networks and increase the data rates, femtocells are designed. Femtocells are small base stations which are aimed to serve home users [3]. Femtocell base stations are cheap devices installed to cover small geographical areas [11]. This will accordingly decrease the macrocell load hence, the number of macrocell base stations (MBSs) required to cover the wireless network will decrease. Also, since femtocells cover small areas, the wireless network capacity will be increased.

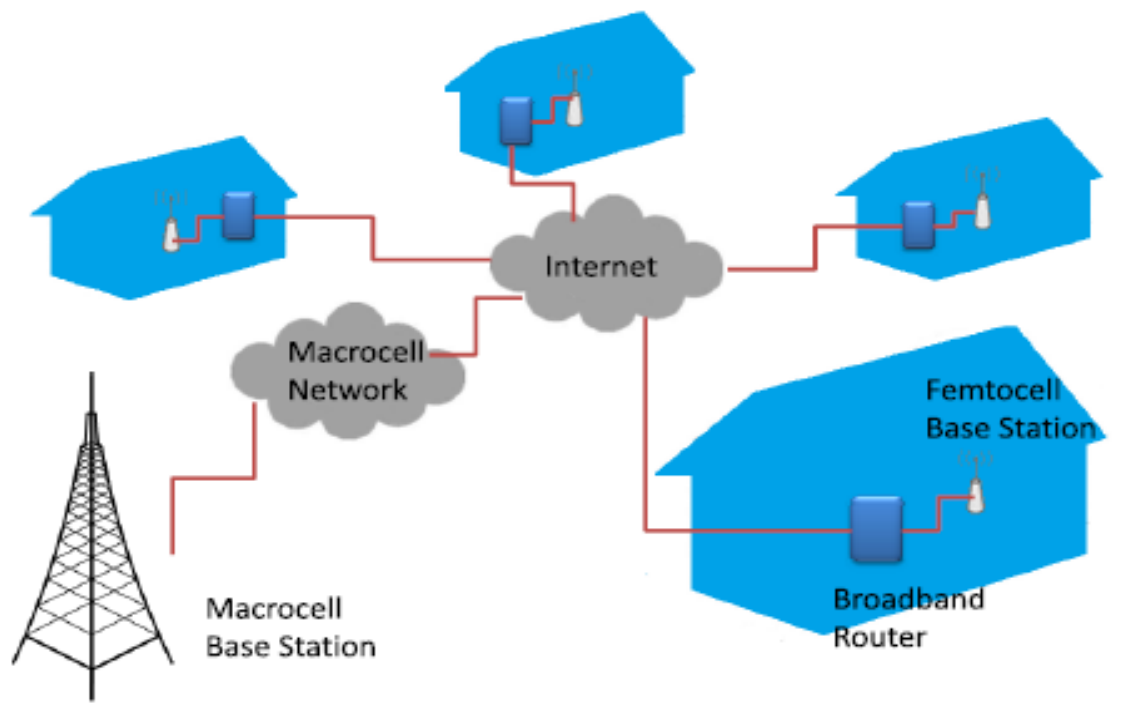

Figure 1-2 Femtocell Network [7] 
Figure 1-2 represents a femtocell network, it's shown in this figure that there is a connection between the base station of the femtocell and the internet via a broadband router. This means that the femtocell users (FUs) is able to use the internet backhaul which has an advantage in reducing the load on the macrocell. Also, the connection between the Femto Base Stations (FBSs) and the internet allows the operation of femtocells in any area where the internet backhaul is available. Even if there is no cellular backhaul, femtocells will be able to provide cellular coverage using the internet backhaul. Femtocells have another advantage in improving the network coverage since they cover a small area. This helps in increasing the QoS especially in rural areas where the indoor network coverage is very poor.

Figure 1-3 shows different types of cells with their corresponding coverage areas [17].

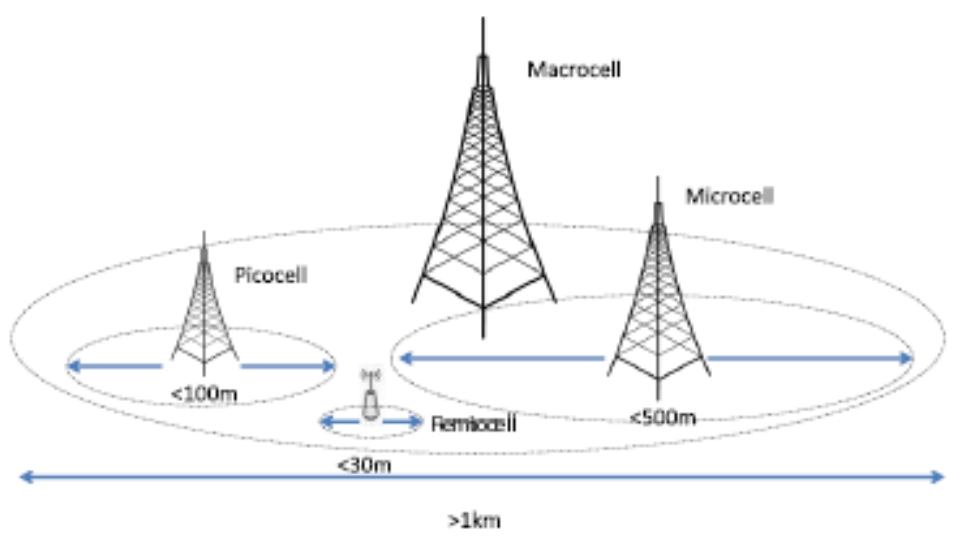

Figure 1-3 Coverage areas of different cell types [7]

In Fig. 1-3, the cells which are compared are the macrocell, microcell, femtocell and picocell. As shown in the figure, femtocell has the smallest coverage area compared to the other cells.

Femtocells have another advantage that they can be purchased and installed by the end user without the need of any infrastructure. This fact makes their construction easy and cheap. While other types of cells such as picocells, microcells and macrocells are not that easy to be constructed and maintained since their construction should be done by the network operator. But, this makes it easy to deal with the interference problem by applying interference management and scheduling methods. While in the case of femtocells, interference management is more complex due to the fact that femtocells are installed anywhere randomly by their users.

Also, the devices of the femtocell users have a longer battery life because the femtocells coverage areas are small hence, the FBSs are operating with a low power and near to their users.

Co-operation between the macrocell and the femtocells is essential by sharing the frequency band since the frequency is an expensive and a limited resource. In Fig. 1-4, different spectrum access types for femtocells are shown. 




Figure 1-4 Femtocells spectrum access types

As shown in the figure, three types of spectrum access are available. These types are dedicated, co-channel and hybrid access [17]. The dedicated access isn't a preferred access type from the point of view of the spectrum efficiency since, in this spectrum access type, the femtocells and the macrocell have different frequency bands. But, on the other side, this reduces the interference that could happen between the two types of cells. The second spectrum access type (co-channel access) allows sharing the same frequency band by the femtocells and the macrocell. This makes the spectrum efficiency high but, high interference could happen hence, intelligent interference management techniques should be applied. In the hybrid spectrum access, macrocell and the femtocells are operating using different frequency bands. But, if the load on the macrocell is increased, macrocell is allowed to share the femtocells frequency bands.

The hybrid spectrum access made the researchers think of giving the macrocell and the femtocells users the permission to access the spectrum which they called open and closed access. The open access allows the access of all base stations regardless it's a MBS or a FBS by all operator subscribers. While the closed access gives the access to a small number of subscribers [15].

In order to maintain high spectrum efficiency, femtocells should share the frequency resources with the macrocell and this will result in a cross-tier interference problem. Due to the fact that femtocells are deployed anywhere randomly within the macrocell, this crosstier interference problem becomes very challenging and intelligent interference management techniques should be applied.

Concerning the uplink interference at the FBSs due to the macrocell users (MUs): If the MU is far away from the MBS, it will send its data with a high power, thus, if at the same time, this MU is near to a FBS, the interference at the FBS will be very high as illustrated in figure 1-5. 




Figure 1-5 Interference in a two-tier network [7] interferer.

When the MU is near to a FBS as shown in Fig. 1-6, it's called a dominant

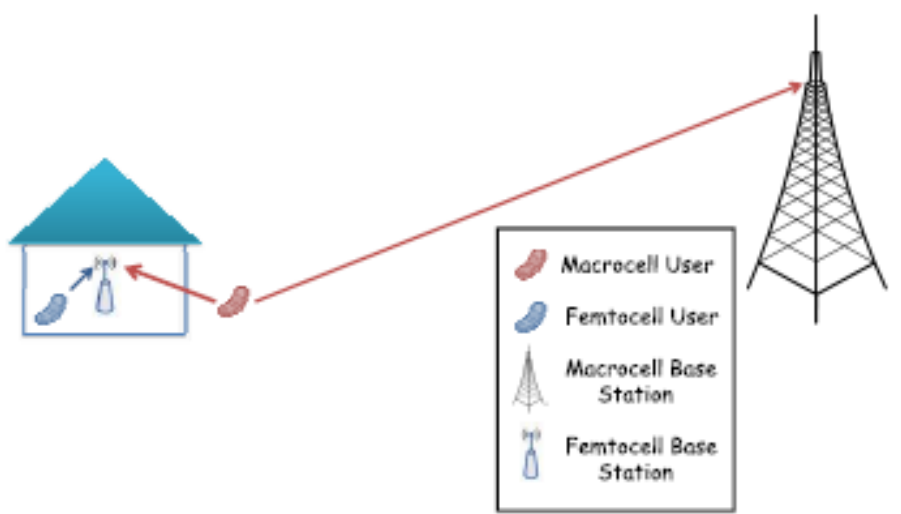

Figure 1-6 Dominant interferer [7]

In [5], interference problem due to macrocell users at the femtocell receivers is addressed. In order to align the interference signals, the macrousers cooperate with the nearest femtocell receivers. Then, Zero Forcing technique is applied to null the aligned interference. In [9], uplink and downlink interference problems in a cellular network with multiple cells are 
addressed and IA is applied. Relays are used to solve the intercell interference and Zero Forcing technique is applied to solve the intracell interference problem. In [6], In order to align the interference, first, the users try to detect the receivers at which they are causing interference then, they design precoders to make the interference signals aligned at the receivers. At each receiver, the users which cause the highest interference are selected to be aligned. In multiuser interference networks, clustering is used to gather the users then, IA technique is applied to these clusters. Clustering is done for networks whose channels are asymmetric. Users which cause high interference are clustered and relatively weak interference can be considered as noise [4],[2]. In heterogeneous networks, the most dominant interference to the macro users is the inter macro user interference and the pico users suffer mainly from the interference due to the macro base station and the inter picocell interference. IA is applied to solve the interference problem where the inter macro user interference is reduced by designing the precoding matrix at the macro base station and interference suppression matrices at the macro users. Pico base stations precoding matrices are formed such that inter picocell interference is aligned with the highest interference coming from the macro base station (cross-layer interference). Interference suppression matrices of the pico users eliminate the aligned interference. In order to decrease the interference at the pico users, power allocation is applied on the macro base station [10],[18]. IA can improve the sum rate in cognitive radio in which the secondary users send their data using the same frequency band of the primary users. At the primary receivers, minimizing the distance between the subspace of the signal received from the secondary transmitters and the interference subspace is also a technique to decrease the interference [12].

\subsection{Interference Alignment (IA)}

In any communication system, the receiver aims to receive the signal transmitted by its desired transmitter only, each receiver tries to get rid of the interference signals coming from the other un-desired transmitters by using the minimum number of dimensions. So, in order to get rid of the interference signals, each receiver aligns all the interferers together in a lower dimensional subspace. Figure 1-7 shows the idea of IA in a 3 users interference channel. 


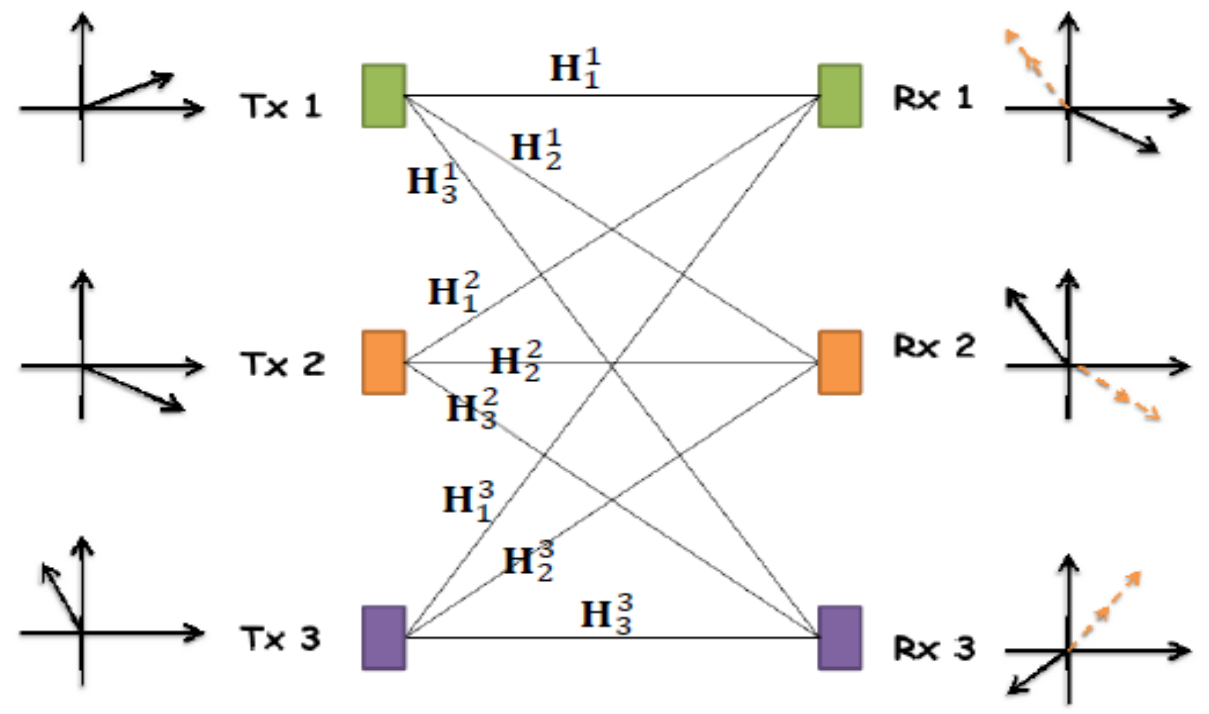

Figure 1-7 Three users interference channel applying IA

As shown in the figure, each receiver aligns two signals which are the interference signals due to the two un-desired transmitters. For instance, receiver 1 aligns the signals coming from transmitters 2 and 3 in a subspace different from its desired signal (transmitted by transmitter 1) subspace. The same concept is applied at receivers 2 and 3.

\subsection{Contribution and Organization}

In this journal paper, the macrocell users that cause high interference on the FBS are selected, for each macrouser, the sum of the square of the channel gains from this macrouser to the FBS is calculated. The average of these sum values is taken as a threshold then, macrocell users whose sum values exceed the threshold are selected. The selected interference signals are then aligned at the FBSs. The aligned signals are suppressed using Minimum Mean Squared Error (MMSE) and Zero Forcing (ZF) suppression techniques. Cumulative Distribution Function (CDF) is used to measure the system performance and compare it to another selection method. The system performance is measured in terms of the Signal to Interference Ratio (SIR) and the system capacity.

In the next section, the system model and its corresponding parameters are presented. In section 3, the proposed interference cancellation algorithm is explained. Section 4 shows the simulations and obtained results. In the last section, the conclusions and the recommended future work are given. 


\section{System model}

The model of the cellular network consists of macro and femto cells. Femto cells are deployed as they cover small geographical areas hence, improve the Qos. In this network, there is only one MBS and a number of FBSs. The uplink of this network is the main focus where the MBS is the secondary receiver (SR) and the FBSs are the primary receivers (PR). $N_{t}$ denotes the number of transmit antennas for each mobile user, $M$ denotes the number of macro users, $F$ is the number of femtocells and $U_{f}$ represents the number of femto users of the fth FBS. There are a number of $N_{o}$ receive antennas at the MBS and $N_{f}$ receive antennas at the $f^{\text {th }}$ FBS. At the $k^{\text {th }}$ FBS, the received signal is expressed as:

$$
\boldsymbol{r}_{k}=\sum_{i=1}^{U_{k}} \sqrt{p_{k i}} \mathbf{H}_{k k}^{i} \mathbf{W}_{k i} \mathbf{s}_{k i}+\sum_{\substack{f=1 \\ f \neq k}}^{F} \sum_{u=1}^{U_{f}} \sqrt{p_{f u}} \mathbf{H}_{k f}^{u} \mathbf{W}_{f u} \mathbf{s}_{f u}+\sum_{m=1}^{M} \sqrt{p_{o m}} \mathbf{H}_{k o}^{m} \mathbf{W}_{o m} \mathbf{s}_{o m}+\mathbf{n}_{k}
$$

where the first term represents the signals coming from the users of the $k^{\text {th }}$ femtocell, the second term represents the signals coming from the users of the other femtocells which is considered as interference, the third term is the interference due to macrocell users and the last term is the noise at the $k^{\text {th }} \mathrm{FBS}$.

$p_{f u}$ is the power transmitted from the $f^{h}$ user in the $u^{\text {th }}$ femtocell. For the same tier, all users are assumed to transmit the same power and this power value is fixed. The channel from the $u^{\text {th }}$ user in the $f^{\text {th }}$ femtocell to the $k^{\text {th }}$ FBS is denoted by $\mathbf{H}_{k f}^{u}$. $\mathbf{W}_{f u}$ is the precoding matrix of the $u^{\text {th }}$ user in the $f^{\text {th }}$ femtocell, $\mathbf{W}_{f u}$ is an $\left(N_{t} \times d\right)$ matrix. Where $d$ is the number of message bits sent by each mobile user and it's assumed to be the same for all users. $\mathbf{s}_{f u}$ denotes the message signal transmitted from the $u^{\text {th }}$ user in the $f^{\text {th }}$ femtocell. $\mathbf{s}_{f u}$ is a $(d \times 1)$ signal.

$p_{o j}$ is the power transmitted from the $j^{\text {th }}$ macro user. The channel from the $m^{\text {th }}$ macro user to the $k^{\text {th }}$ FBS is represented by $\mathbf{H}_{k o}^{m}$. While, $\mathbf{W}_{o m}$ is an $\left(N_{t} \times d\right)$ matrix which represents the precoding matrix of the $m^{\text {th }}$ macro user. The message signal of the $m^{\text {th }}$ macro user is represented by $\mathbf{s}_{o m}$ and it's a $(d \times 1)$ message signal.

$\mathbf{n}_{k}$ is composed of independent Gaussian random variables with zero mean, for this noise, $E\left\{\mathbf{n}_{k} \mathbf{n}_{k}{ }^{H}\right\}=\sigma^{2} \mathbf{I}$.

Any transmitted message signal element of $\mathbf{s}_{f u}$ or $\mathbf{s}_{o m}$ belongs to the following set: $\{+1,-1\}$ and is chosen randomly with equal probability where $f=1, \ldots, F, u=1, \ldots, U_{f}$ and $m=$ $1, \ldots, M . p_{f u}$ is the power transmitted from the $f^{\text {th }}$ user in the $u^{\text {th }}$ femtocell and $p_{o j}$ is the power transmitted from the $j^{\text {th }}$ macro user. For the same tier, all users are assumed to transmit the same power and this power value is fixed. For all mobile users, the precoding matrices satisfy $\operatorname{tr}\left(\mathbf{W}_{o j}^{H} \mathbf{W}_{o j}\right)=\operatorname{tr}\left(\mathbf{W}_{k u}^{H} \mathbf{W}_{k u}\right)=1$ where $j$ belongs to the following set $\{1, \ldots, M\}, k$ belongs to $\{1, \ldots, F\}$ and $u$ belongs to $\left\{1, \ldots, U_{k}\right\}$. $\operatorname{tr}(\mathbf{A})$ represents the trace of a square matrix $\mathbf{A}$ which is defined as the sum of the elements on the main diagonal. 
Figure 2-1 is an illustration figure of a system model with two MUs and two femtocells each contains two FUs. In this figure, the dashed arrows represent the channels from unintended transmitters which are considered as interferers. While solid arrows are the channels between the femtousers and their desired receivers.



Figure 2-1 Channel model for two macrocell users and two femtocells with two femtousers per each cell

\section{Proposed Interference cancellation algorithm}

The interference cancellation algorithm is done through three phases. The first phase is the selection of the macrocell users which will be aligned at the femto base stations. Afterwards, the selected interference signals will be aligned together at the femto base stations. The third phase is the suppression of the aligned interferers. The three phases are explained in sections 3.1, 2 and 3.3 respectively. 


\subsection{Proposed Macrocell Users Selection}

In this section, the selection method of the macrocell users which will be aligned at the femtocell receivers is explained. The ideal scenario is to align all the macrocell users at each femto receiver in order to solve the interference problem but, this couldn't be done since the number of antennas at the femtocell receiver limits the maximum number of the signals which will be aligned. Therefore, some macrocell users should be selected from the total macrocell users to be aligned at the femto receivers. This selection is done as follows: First, at each femto receiver, the sum of the square of the channels gains from each macro user to this receiver is calculated and the average of those sum values is calculated and taken as a threshold. This threshold yields the alignment of the least number of interference signals to maintain the system performance with a low complexity. The channel from the $m^{\text {th }}$ macro user to the $k^{\text {th }}$ FBS is represented by $\mathbf{H}_{k o}^{m}=\left[\begin{array}{ccc}\mathrm{h}_{11} & \cdots & \mathrm{h}_{1 N_{t}} \\ \vdots & \ddots & \vdots \\ \mathrm{h}_{N_{k} 1} & \cdots & \mathrm{h}_{N_{k} N_{t}}\end{array}\right]$, where $N_{t}$ denotes the number of transmit antennas for each mobile user and $N_{k}$ receive antennas at the $k^{\text {th }}$ FBS. Then, the sum of the square of the channels gains from macro user $m$ to femto receiver $k$ is represented by $\mathrm{M}_{m}$ where $\mathrm{M}_{m}=\operatorname{tr}\left(\left(\mathbf{H}_{k o}^{m}\right)^{H} \mathbf{H}_{k o}^{m}\right)$. The threshold is the average of all $\mathrm{M}_{m}$ where $m=1, \ldots, M$ where $M$ is the number of macrocell users. For each macro user, $\mathrm{M}_{m}$ is compared to the threshold and macrocell users whose $\mathrm{M}_{m}$ are greater than the threshold are selected to be aligned at femto receiver $k$. An example is given in figure 3-1 in which the macrouser is equipped with two transmit antennas and the femto receiver receives the signals using three receive antennas. The channel matrix from this macrouser and FBS is $\mathbf{H}_{k o}^{m}=\left[\begin{array}{ll}\mathrm{h}_{11} & \mathrm{~h}_{12} \\ \mathrm{~h}_{21} & \mathrm{~h}_{22} \\ \mathrm{~h}_{31} & \mathrm{~h}_{32}\end{array}\right]$. Since $\mathrm{M}_{m}=\operatorname{tr}\left(\left(\mathbf{H}_{k o}^{m}\right)^{H} \mathbf{H}_{k o}^{m}\right)$ therefore, $\mathrm{M}_{m}=\mathrm{h}_{11}{ }^{2}+\mathrm{h}_{21}{ }^{2}+\mathrm{h}_{31}{ }^{2}+$ $\mathrm{h}_{12}{ }^{2}+\mathrm{h}_{22}{ }^{2}+\mathrm{h}_{32}{ }^{2}$. And since the threshold $T$ is the average of all $\mathrm{M}_{m}$ where $m=1, \ldots, M$ therefore, the threshold is calculated as follows: $T=\frac{\sum_{m=1}^{m=M} \mathrm{M}_{m}}{M}$. If $\mathrm{M}_{m}>T$, the macrouser $m$ will be selected to be aligned at the femto receiver $k$.

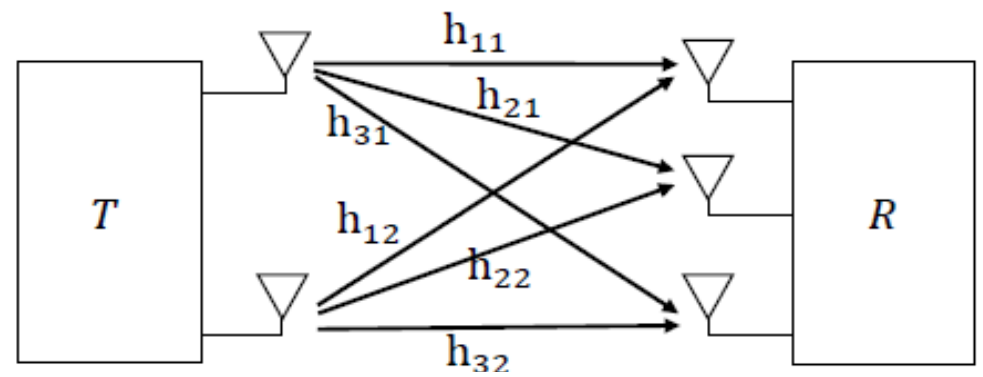

Figure 3-1 Channel model for macrouser equipped with two transmit antennas and femto receiver with three receive antennas 


\subsection{Cross-tier Interference Alignment}

The second step is to align the selected interference signals at each FBS. So, at each FBS, we define the subspace which the selected interference signals will span. Note that at each FBS, all the selected interference signals must span the same subspace in order to be aligned. The basis of the subspaces chosen for alignment at each PR are represented by the columns of the matrices $\mathbf{V}_{1}, \mathbf{V}_{2}, \ldots, \mathbf{V}_{F}$. Hence, each column of $\mathbf{H}_{k o}^{j} \mathbf{W}_{o j}$ is a linear combination of the columns of $\mathbf{V}_{k}$ where $k$ is the FBS at which the interference will be aligned and $j$ belongs to the selected interferers set [7] .

Let $\mathbf{W}_{o j}=\left[\mathbf{w}_{o j}^{1} \mathbf{w}_{o j}^{2} \ldots . \mathbf{w}_{o j}^{d}\right]$ and $\mathbf{V}_{k}=\left[\mathbf{v}_{k}^{1} \mathbf{v}_{k}^{2} \ldots . \mathbf{v}_{k}^{d}\right]$ where the $i^{\text {th }}$ column of $\mathbf{W}_{o j}$ is denoted by $\mathbf{w}_{o j}^{i}$ and the $i^{\text {th }}$ column of $\mathbf{V}_{k}$ is denoted by $\mathbf{v}_{k}^{i}$. So, the span of the interference signals could be represented by $\mathbf{H}_{k o}^{j} \mathbf{w}_{o j}^{i}=\left[\alpha_{k j}^{i} \mathbf{v}_{k}^{1}+\beta_{k j}^{i} \mathbf{v}_{k}^{2}+\cdots+\theta_{k j}^{i} \mathbf{v}_{k}^{d}\right]$ where $\alpha_{k j}^{i}, \beta_{k j}^{i}$ and $\theta_{k j}^{i}$ are scalars and $i=1, \ldots, d$. The previous equation can be re-written as follows:

$$
\begin{gathered}
\mathbf{H}_{k o}^{j} \mathbf{w}_{o j}^{1}=\left[\alpha_{k j}^{1} \mathbf{v}_{k}^{1}+\beta_{k j}^{1} \mathbf{v}_{k}^{2}+\cdots+\theta_{k j}^{1} \mathbf{v}_{k}^{d}\right] \\
\mathbf{H}_{k o}^{j} \mathbf{w}_{o j}^{2}=\left[\alpha_{k j}^{2} \mathbf{v}_{k}^{1}+\beta_{k j}^{2} \mathbf{v}_{k}^{2}+\cdots+\theta_{k j}^{2} \mathbf{v}_{k}^{d}\right] \\
\cdot \\
\cdot \\
\mathbf{H}_{k o}^{j} \mathbf{w}_{o j}^{d}=\left[\alpha_{k j}^{d} \mathbf{v}_{k}^{1}+\beta_{k j}^{d} \mathbf{v}_{k}^{2}+\cdots+\theta_{k j}^{d} \mathbf{v}_{k}^{d}\right]
\end{gathered}
$$

Equation 3.1 can be expressed as linear matrix equations as:

$$
\widetilde{\mathbf{H}}_{k j} \mathbf{w}_{o j}=\widetilde{\mathbf{A}}_{k j} \mathbf{v}_{k}
$$

Where $d$ blocks of $\mathbf{H}_{k o}^{j}$ form the block diagonal matrix $\widetilde{\mathbf{H}}_{k j}$ as follows:

$$
\widetilde{\mathbf{H}}_{k j}=\left[\begin{array}{cccc}
\mathbf{H}_{k o}^{j} & 0 & \cdots & 0 \\
0 & \mathbf{H}_{k o}^{j} & \cdots & 0 \\
\vdots & & \ddots & \vdots \\
0 & \cdots & 0 & \mathbf{H}_{k o}^{j}
\end{array}\right]
$$


And

$$
\mathbf{w}_{o j}=\left[\left(\mathbf{w}_{o j}^{1}\right)^{T}\left(\mathbf{w}_{o j}^{2}\right)^{T} \ldots\left(\mathbf{w}_{o j}^{d}\right)^{T}\right]^{T}
$$

Let a matrix $\mathbf{A}_{k j}$ be represented as in the following equation:

$$
\mathbf{A}_{k j}=\left[\begin{array}{cccc}
\alpha_{k j}^{1} & \beta_{k j}^{1} & \cdots & \theta_{k j}^{1} \\
\vdots & \vdots & \ddots & \vdots \\
\alpha_{k j}^{d} & \beta_{k j}^{d} & \cdots & \theta_{k j}^{d}
\end{array}\right]
$$

Then, the matrix of coefficients $\widetilde{\mathbf{A}}_{k j}$ is as follows:

$$
\widetilde{\mathbf{A}}_{k j}=\mathbf{A}_{k j} \otimes \mathbf{I}_{N_{f} \times N_{f}}
$$

where $\mathbf{I}_{N_{f} \times N_{f}}$ is the identity matrix of dimensions $N_{f} \times N_{f}$ and $\otimes$ denotes the Kronecker product

Finally, $\mathbf{v}_{k}=\left[\left(\mathbf{v}_{k}^{1}\right)^{T}\left(\mathbf{v}_{k}^{2}\right)^{T} \ldots\left(\mathbf{v}_{k}^{d}\right)^{T}\right]^{T}$.

Then, the interference alignment is done using the algorithm described in Table 3.1:

Table 3.1: Interference Alignment Algorithm

\section{Indices Steps}

1 The matrices $\mathbf{A}_{k j}$ and $\mathbf{V}_{1}, \mathbf{V}_{2}, \ldots, \mathbf{V}_{F}$ are initialized where $k=1, \ldots, F$ and $j=1, \ldots$, $M$. The initialized elements are taken independently from the standard normal distribution with zero mean and a unit variance.

2 The vectors $\mathbf{w}_{o 1}, \mathbf{w}_{o 2}, \ldots, \mathbf{w}_{o M}$ are formed according to the following:

$\mathbf{w}_{o j}=\underset{\mathbf{w}_{o j}}{\operatorname{argmin}} \sum_{k \in A_{j}}\left\|\widetilde{\mathbf{H}}_{k j} \mathbf{w}_{o j}-\widetilde{\mathbf{A}}_{k j} \mathbf{v}_{k}\right\|^{2}$, where $A_{j}$ is the set of PRs at which the ${ }^{\text {th }}$ SU will be aligned.

These vectors must also satisfy the following: $\operatorname{tr}\left(\left(\mathbf{w}_{o j}\right)^{H} \mathbf{w}_{o j}\right)=1$, so that each SU has a transmit power which equals $\operatorname{tr}\left(\left(\sqrt{p_{o j}} \mathbf{w}_{o j}\right)^{H} \sqrt{p_{o j}} \mathbf{w}_{o j}\right)=p_{o j}$.

3 The precoding matrices $\mathbf{W}_{o 1}, \mathbf{W}_{o 2}, \ldots . \mathbf{W}_{o M}$ are formed using the vectors $\mathbf{w}_{O 1}, \mathbf{w}_{O 2}, \ldots . \mathbf{w}_{O M}$. 
4

The vectors $\mathbf{v}_{1}, \mathbf{v}_{2}, \ldots, \mathbf{v}_{F}$ are determined by fixing the precoding matrices and applying the following equation: $\mathbf{v}_{k}=\underset{\mathbf{v}_{k}}{\operatorname{argmin}} \sum_{j \in S_{k}}\left\|\widetilde{\mathbf{H}}_{k j} \mathbf{w}_{o j}-\widetilde{\mathbf{A}}_{k j} \mathbf{v}_{k}\right\|^{2}$, where $S_{k}$ is the selected secondary users at primary receiver $k$.

5 For $k=1, \ldots, F$ and $j=1, \ldots, M$, the matrix $\mathbf{A}_{k j}$ is constructed as follows: First, the following equation is formed for a given $\mathbf{H}_{k o}^{j}, \mathbf{W}_{o j}$ and $\mathbf{V}_{k}$ :

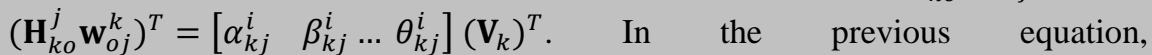
$\left[\begin{array}{llll}\alpha_{k j}^{i} & \beta_{k j}^{i} & \ldots & \theta_{k j}^{i}\end{array}\right]=\left(\mathbf{a}_{k j}^{i}\right)^{T}$ so, $\mathbf{a}_{k j}^{i}=\mathbf{V}_{k}^{\dagger} \mathbf{H}_{k o}^{j} \mathbf{w}_{o j}^{i}$ and $\mathbf{V}_{k}^{\dagger}=\left(\mathbf{V}_{k}^{H} \mathbf{V}_{k}\right)^{-1} \mathbf{V}_{k}^{H}$. $\mathbf{A}_{k j}^{T}=\left[\begin{array}{llll}\mathbf{a}_{k j}^{1} & \mathbf{a}_{k j}^{2} & \ldots & \mathbf{a}_{k j}^{d}\end{array}\right]$.

Repeat the previous steps (from step 2 to step 5) until convergence.

Convergence is measured by the total leaked interference from all the PRs and SUs denoted by $L$.

In step 6 in the previous table, $L$ can be expressed by the following equation:

$$
L=\sum_{j=1}^{M} \sum_{k \in A_{j}}\left\|\widetilde{\mathbf{H}}_{k j} \mathbf{w}_{o j}-\widetilde{\mathbf{A}}_{k j} \mathbf{v}_{k}\right\|^{2}=\sum_{k=1}^{F} \sum_{j \in S_{k}}\left\|\widetilde{\mathbf{H}}_{k j} \mathbf{w}_{o j}-\widetilde{\mathbf{A}}_{k j} \mathbf{v}_{k}\right\|^{2}
$$

$L$ is decreased after each iteration, until it stopes to be decreased. In section 4 , it will be shown that $L$ almost reaches its minimum value (zero) in a limited number of iterations. Hence, convergence occurs.

This cross-tier IA algorithm is a distributed algorithm because each MU requires to know the channel between it and all the receivers which is causing interference at them. This channel knowledge is essential so that the MU can design its precoding vector. In order to apply the alignment, the MU needs to know $\widetilde{\mathbf{A}}_{k j} \mathbf{v}_{k}$ as well.

\subsection{Precoders and Decoders Design for Femtocells}

For the primary users, the precoding and decoding matrices are formed based on different techniques. If $\widehat{\mathbf{s}}_{k j}$ denotes the estimated signal for user $j$ in the $k^{\text {th }}$ femtocell and $\mathbf{G}_{k j}$ denotes the decoding matrix of this user, then $\hat{\mathbf{s}}_{k j}$ can be represented as follows: 


$$
\begin{gathered}
\hat{\mathbf{s}}_{k j}=\sum_{i=1}^{U_{k}} \sqrt{p_{k i}}\left(\mathbf{G}_{k j}\right)^{H} \mathbf{H}_{k k}^{i} \mathbf{W}_{k i} \mathbf{s}_{k i}+\sum_{\substack{f=1 \\
f \neq k}}^{F} \sum_{u=1}^{U_{f}} \sqrt{p_{f u}}\left(\mathbf{G}_{k j}\right)^{H} \mathbf{H}_{k f}^{u} \mathbf{W}_{f u} \mathbf{s}_{f u} \\
+\sum_{m=1}^{M} \sqrt{p_{o m}}\left(\mathbf{G}_{k j}\right)^{H} \mathbf{H}_{k o}^{m} \mathbf{W}_{o m} \mathbf{s}_{o m}+\left(\mathbf{G}_{k j}\right)^{H} \mathbf{n}_{k}
\end{gathered}
$$

The interference due to other femtocells is treated as noise for simplicity, this noise is as follows:

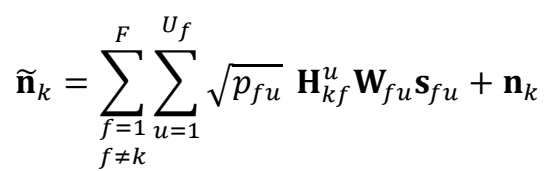

Subsections 3.3.1 and 3.3.2 explain the two techniques used to design the precoders and decoders of the femtocells.

\subsubsection{Minimum Mean Squared Error (MMSE) based Precoders and Decoders Design}

For the primary users, the precoders and decoders are designed based on the Minimum Mean Squared Error (MMSE) technique. For the $k^{\text {th }} \mathrm{PR}$, the sum mean squared error is as follows:

$$
\mathrm{MSE}=\sum_{j=1}^{U_{k}} E\left\{\left\|\hat{\mathbf{s}}_{k j}-\mathbf{s}_{k j}\right\|^{2}\right\}
$$

Let $\mathbf{R}_{\tilde{n}}$ be the covariance matrix of $\widetilde{\mathbf{n}}_{k}$ (the interference due to other femtocells) then, according to [13], the decoding and precoding matrices are as follows:

$$
\begin{gathered}
\mathbf{G}_{k j}=\left(\sum_{i=1}^{U_{k}} p_{k i} \mathbf{H}_{k k}^{i} \mathbf{W}_{k i}\left(\mathbf{H}_{k k}^{i} \mathbf{W}_{k i}\right)^{H}+\sum_{m=1}^{M} p_{o m} \mathbf{H}_{k o}^{m} \mathbf{W}_{o m}\left(\mathbf{H}_{k o}^{m} \mathbf{W}_{o m}\right)^{H}\right. \\
\left.+\mathbf{R}_{\tilde{n}}\right)^{-1} \sqrt{p_{k j}} \mathbf{H}_{k k}^{j} \mathbf{W}_{k j}
\end{gathered}
$$




$$
\mathbf{W}_{k j}=\left(\sum_{i=1}^{U_{k}} p_{k i}\left(\mathbf{H}_{k k}^{j}\right)^{H} \mathbf{G}_{k i}\left(\mathbf{G}_{k i}\right)^{H} \mathbf{H}_{k k}^{j}+\mu_{k j} \mathbf{I}\right)^{-1} \sqrt{p_{k j}}\left(\mathbf{H}_{k k}^{j}\right)^{H} \mathbf{G}_{k j}
$$

Where $j=1, \ldots, U_{k}$ and $\mu_{k j}$ is determined to satisfy the following condition $\operatorname{tr}\left(\left(\mathbf{W}_{k j}\right)^{H} \mathbf{W}_{k j}\right)$ $=1$ so that the femtocell users transmission power satisfy $\operatorname{tr}\left(\left(\sqrt{p_{k j}} \mathbf{W}_{k j}\right)^{H} \sqrt{p_{k j}} \mathbf{W}_{k j}\right)=p_{k j}$.

\subsubsection{Zero Forcing (ZF) based Precoders and Decoders Design}

When ZF is applied on the aligned interference signals, the interference is nulled at the receivers. Let $E\left\{\tilde{\mathrm{n}}_{\mathrm{k}}\left(\tilde{\mathrm{n}}_{\mathrm{k}}\right)^{\mathrm{H}}\right\}=\tilde{\sigma}^{2} \mathbf{I}$ and let $\left[\mathbf{B}_{k}^{0} \mathbf{B}_{k}^{1}\right] \Lambda_{k} \mathbf{D}_{k}$ be the singular-value decomposition (SVD) of $\mathbf{H}_{k o}^{1} \mathbf{W}_{o 1}$. Then, $\left(\mathbf{B}_{k}^{0}\right)^{H} \mathbf{H}_{k k}^{j}=\widehat{\mathbf{H}}_{k k}^{j}$. According to [5], the decoding and precoding matrices in which the $\mathrm{ZF}$ technique is applied are expressed as follows:

$$
\begin{gathered}
\mathbf{G}_{k j}=\left(\sum_{i=1}^{U_{k}}\left(\widehat{\mathbf{H}}_{k k}^{i} \mathbf{W}_{k i}\right)\left(\widehat{\mathbf{H}}_{k k}^{i} \mathbf{W}_{k i}\right)^{H}+\tilde{\sigma}^{2} \mathbf{I}\right)^{-1} \widehat{\mathbf{H}}_{k k}^{j} \mathbf{W}_{k j} \\
\mathbf{W}_{k j}=\left(\sum_{i=1}^{U_{k}}\left(\widehat{\mathbf{H}}_{k k}^{j}\right)^{H} \mathbf{G}_{k i}\left(\mathbf{G}_{k i}\right)^{H} \widehat{\mathbf{H}}_{k k}^{j}+\mu_{k j} \mathbf{I}\right)^{-1}\left(\widehat{\mathbf{H}}_{k k}^{j}\right)^{H} \mathbf{G}_{k j}
\end{gathered}
$$

Where $j=1, \ldots, U_{k}$ and $\mu_{k j}$ is determined to satisfy the following condition $\operatorname{tr}\left(\left(\mathbf{W}_{k j}\right)^{H} \mathbf{W}_{k j}\right)$ $=1$ so that the femtocell users transmission power satisfy $\operatorname{tr}\left(\left(\sqrt{p_{k j}} \mathbf{W}_{k j}\right)^{H} \sqrt{p_{k j}} \mathbf{W}_{k j}\right)=p_{k j}$.

\section{Simulations and Results}

The performance of the proposed IA algorithms is measured in terms of the SIR (calculated in $\mathrm{dB}$ ), where the interference here refers to the cross-tier interference due to the macrocell users at the FBSs. SIR is calculated in $\mathrm{dB}$ by $10 \log _{10}(1+S / I)$. Where $S$ is the desired 
signal power (measured in Watts) and $I$ is the interference signal power (measured in Watts).

Also, the capacity is used to measure the system performance. The capacity represents the highest rate at which information can be reliably transmitted. Usually, the performance is evaluated by the Bit Error Rate (BER). The BER is defined as the ratio between the number of incorrect bits to the total number of transmitted bits. In our simulations, the performance is measured using Cumulative Distribution Function (CDF). Where the CDF of a realvalued random variable $\mathrm{X}$ evaluated at $\mathrm{X}$, is the probability that $\mathrm{X}$ will take a value less than or equal to $\mathrm{x}$

The results are compared to another user selection method which aligns all the possible interferers. In other words, the user selection method which our results are compared to aligns all the interference signals that are causing the highest effect at the femto receiver. But, if the number of those interference signals exceeds the number of receive antennas, interference signals are arranged in a descending order then, the alignment is done for a number of interference signals equals to the number of receive antennas taken from the descending order starting from the highest interferer.

In this work, the simulations parameters are as shown in the following table.

Table 4.1: Simulations Parameters

\begin{tabular}{|l|l|}
\hline Number of femtocells & 3 \\
\hline Number of femtousers/cell & 2 \\
\hline Number of macrocell users & 6 \\
\hline Number of transmit antennas & 2 \\
\hline Number of receive antennas & 4 \\
\hline Message length & 1 bit \\
\hline Femtouser transmit power & 0 dBm \\
\hline Macrouser transmit power & 10 dBm \\
\hline Channels & Rayleigh fading channels \\
\hline
\end{tabular}


Hence, our system model can be represented as in Fig. 4-1. In this figure, at any FBS, the solid arrows represent the desired signal transmitted by its femtocell users. While, the interference due to the other femtocells is represented by the dash-dot arrows. Dashed arrows represent the cross-tier interference signals due to the macrocell users. 


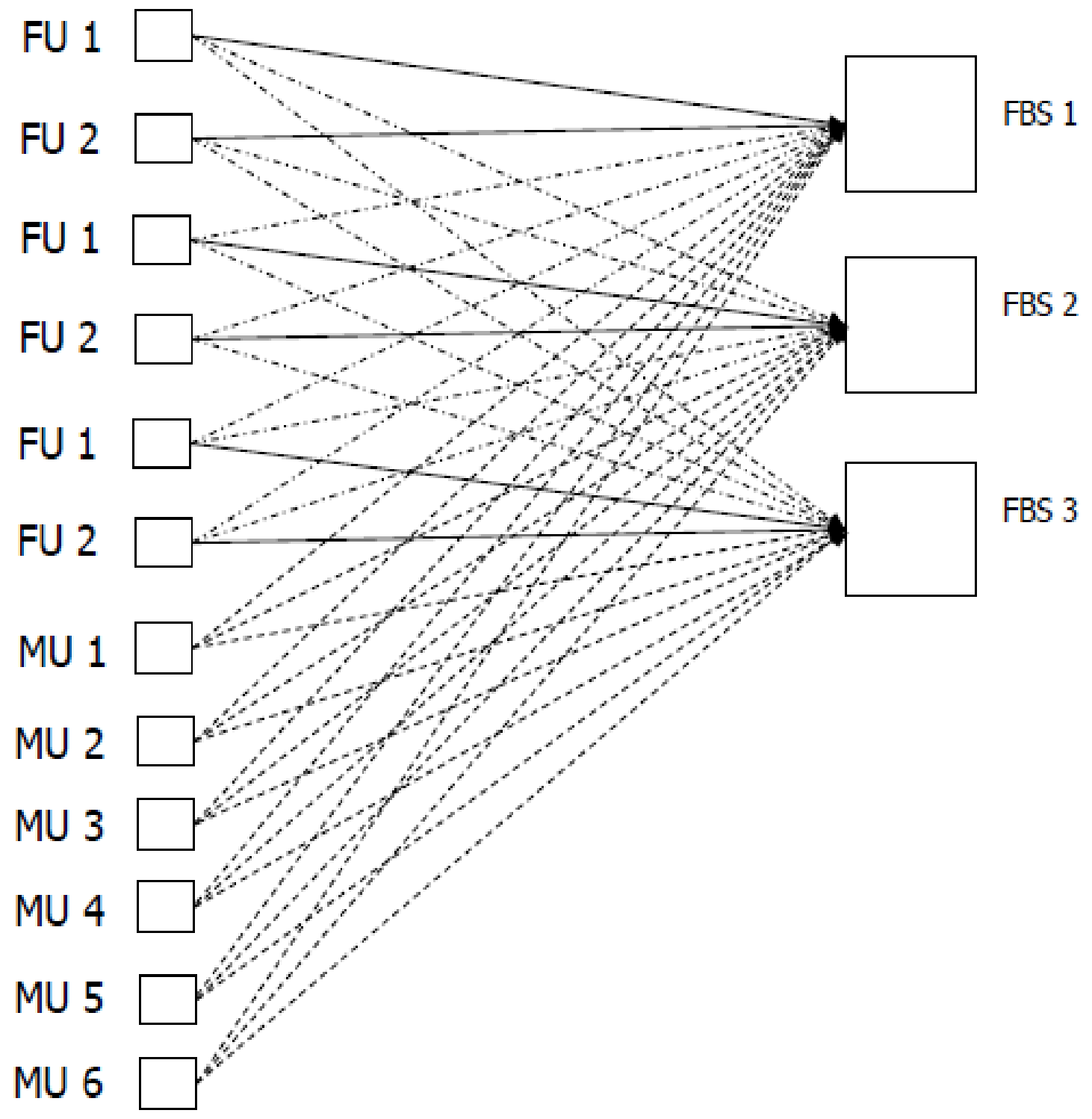

Figure 4-1 System model used in the simulations 
Fig. 4-2 shows the convergence of the proposed selective IA algorithm applied on the macro users. It is shown from this figure that as the number of iterations increases, the total leaked interference decreases until convergence occurs. At the fourth iteration, the total leaked interference is $0.1449 \mathrm{~mW}$ which is very close to zero.

According to the IA algorithm applied in this paper to align the macrocell users signals at the FBSs, when we fix $\mathbf{v}_{1}, \ldots \ldots, \mathbf{v}_{F}$ and tried to get $\mathbf{w}_{o 1}, \ldots . \mathbf{w}_{O M}$ as step 2 in the algorithm represented by table 3.1, the value of $L$ is decreased according to the first term in equation (3.7). The same scenario is repeated when $\mathbf{w}_{o 1}, \ldots \mathbf{w}_{o M}$ are fixed and step 4 in table 3.1 is applied to get $\mathbf{v}_{1}, \ldots \ldots, \mathbf{v}_{F}$ and the value of the $\mathrm{L}$ is decreased according to the second term in equation (3.7). Hence, after each iteration L is decreased, and since it's bounded below by zero, convergence occurs.

Our numerical results show that the leakage interference converges to a value very close to zero as shown in Fig. 4-2. This convergence happens after a number of four iterations only.

Fig. 4-3 shows the CDF of the SIR using the highest selection IA algorithm applying MMSE technique to suppress the aligned interference signals. As shown in the figure, when IA is applied, the SIR is increased and accordingly the system performance is improved.

The system capacity is measured using CDF for the cases when the highest selection IA algorithm applying MMSE technique is applied and when no IA algorithm is used in Fig. 44. The results show that the system capacity which is calculated as $\log _{2}(1+S / I)$ is improved when IA is applied.



Figure 4-2 Convergence of the proposed IA algorithm 


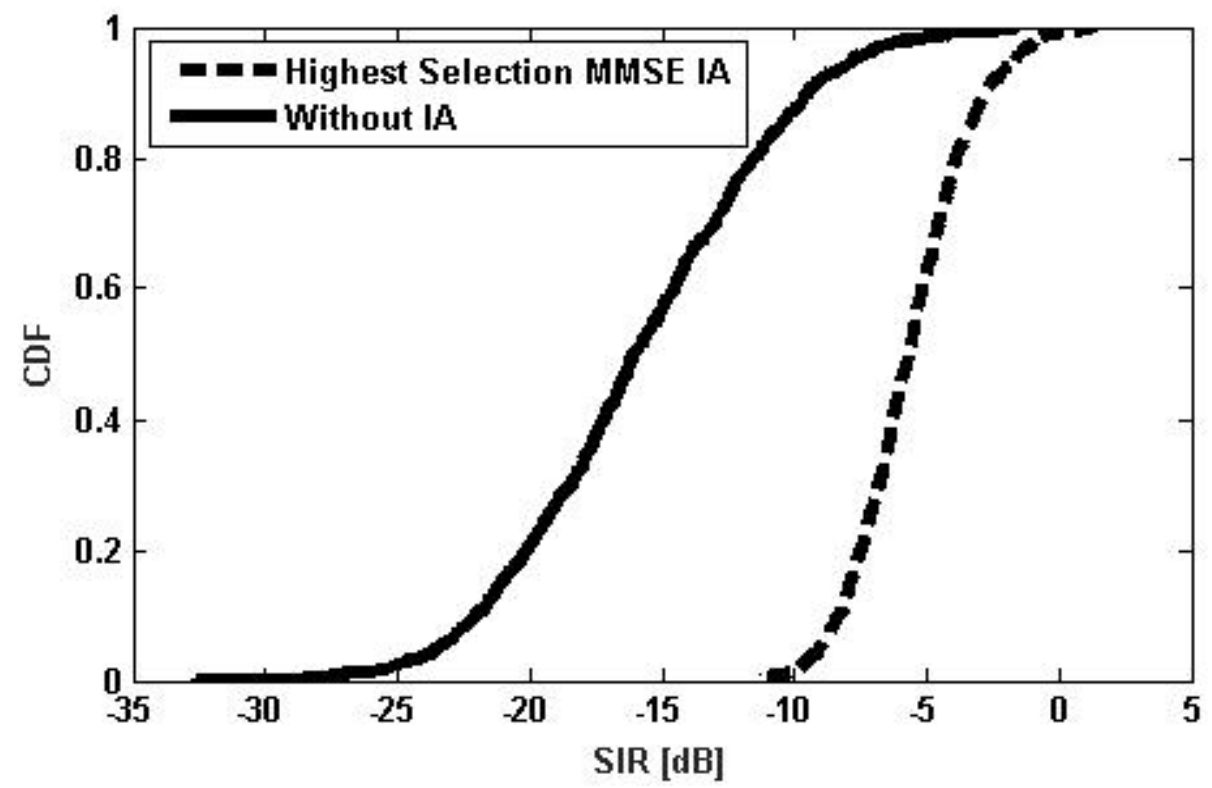

Figure 4-3 CDF of the signal to interference ratio using the highest selection IA

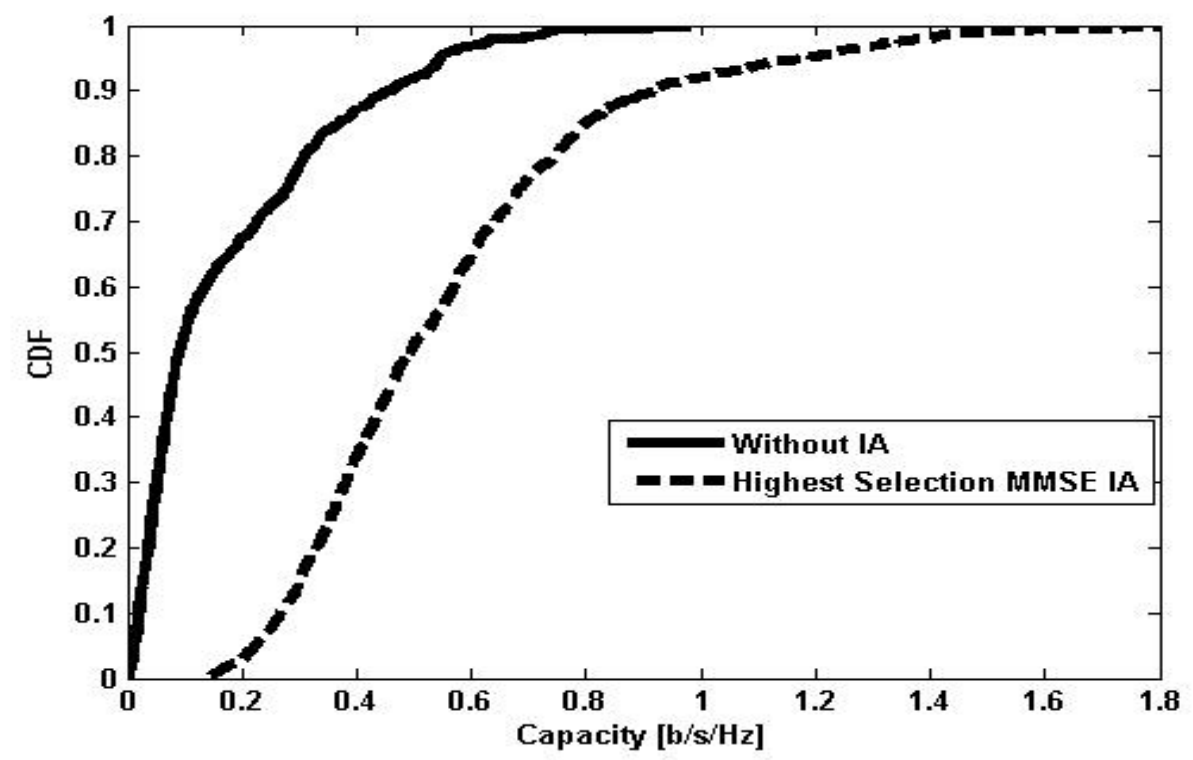

Figure 4-4 CDF of the capacity using the highest selection IA 
In Fig. 4-5, the CDF of the SIR using the proposed average selection IA algorithm applying MMSE technique to suppress the aligned interference signals is shown. It's clear in this figure that when the proposed IA is applied, the SIR is increased. For instance, at CDF $=0.5$, the SIR is increased by around $10 \mathrm{~dB}$.

Fig. 4-6 represents the system capacity when the proposed average selection IA algorithm applying MMSE technique to suppress the aligned interference signals is applied and when no IA is applied measured by CDF. As shown in the figure, there is an increase in the system capacity when the proposed IA is applied. This increase is around $0.2 \mathrm{~b} / \mathrm{s} / \mathrm{Hz}$ at $\mathrm{CDF}=0.5$.

The CDF of the Signal to Interference Ratio by using the average selection IA applying Zero Forcing technique is shown in Fig. 4-7. SIR is increased significantly when this proposed IA method is applied. That's because ZF nulls the interference signals at the femto receivers. For example at $\mathrm{CDF}=0.5$, SIR using the proposed IA method is approximately 0 $\mathrm{dB}$ while, without IA, SIR is around $-18 \mathrm{~dB}$. This means that SIR is increased by around 18 dB.

Fig. 4-8 presents the capacity measured by CDF by using the average selection IA applying Zero Forcing technique. The proposed IA increased the system capacity as shown in the figure. At $\mathrm{CDF}=0.5$, this increase is around $0.4 \mathrm{~b} / \mathrm{s} / \mathrm{Hz}$.

Fig. 4-9 compares between the proposed IA algorithms in terms of the SIR. As shown in the figure, applying Zero Forcing Suppression technique results in higher SIR values than that of MMSE suppression technique. Zero Forcing has better performance because it nulls the aligned interference signals. It's also clear in the figure that the SIR values of the average selection IA are very close to the SIR values of the highest selection IA applying the same suppression technique. Average selection IA has lower SIR values than that of the highest selection IA because it aligns less number of interference signals. So, average selection IA is less complex than the highest selection IA due to the fact that it aligns less interferers. Hence, the performance when applying the average selection IA is very close to the highest selection IA and with a lower complexity which is the advantage of applying the average selection IA.

In Fig. 4-10, the system capacity when applying the proposed IA algorithms is compared using CDF. The results show that ZF results in higher capacities than MMSE suppression technique. Moreover, when applying the same suppression technique on the average selection IA and the highest selection IA, the capacity values are very close to each other. 


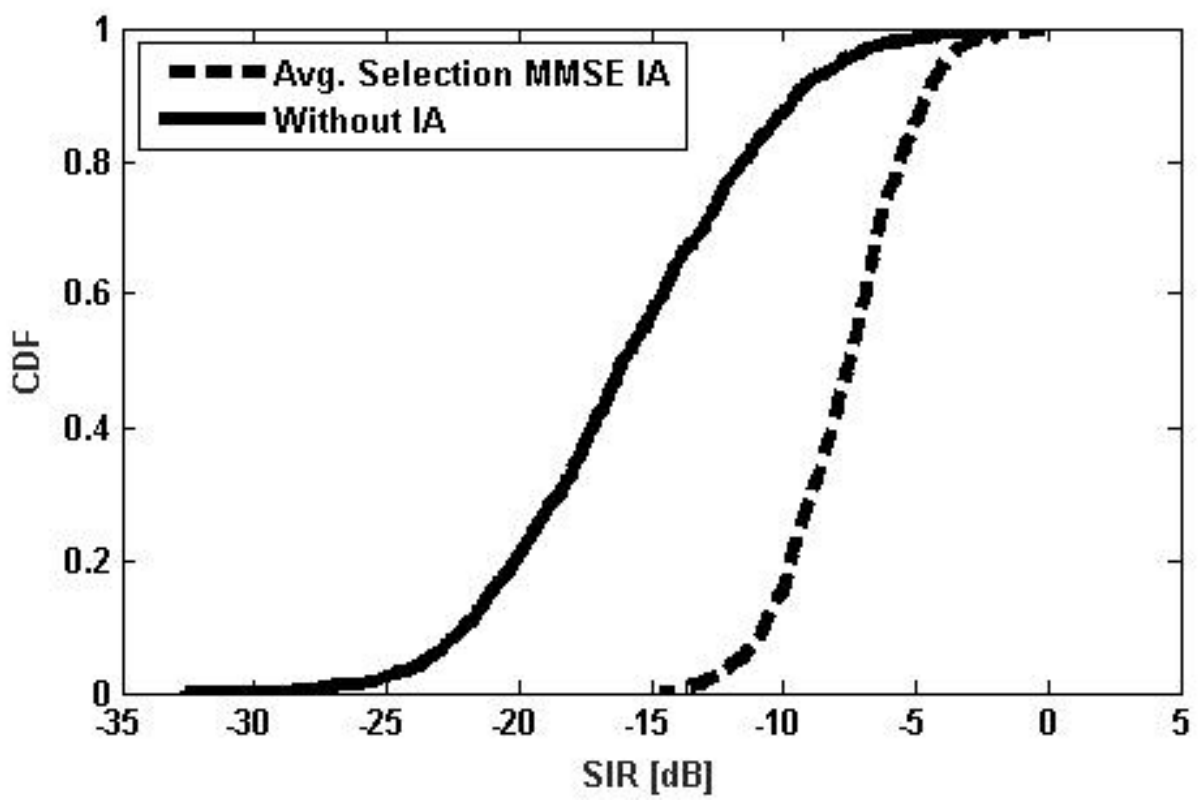

Figure 4-5 CDF of the s ignal to interference ratio using the average selection IA

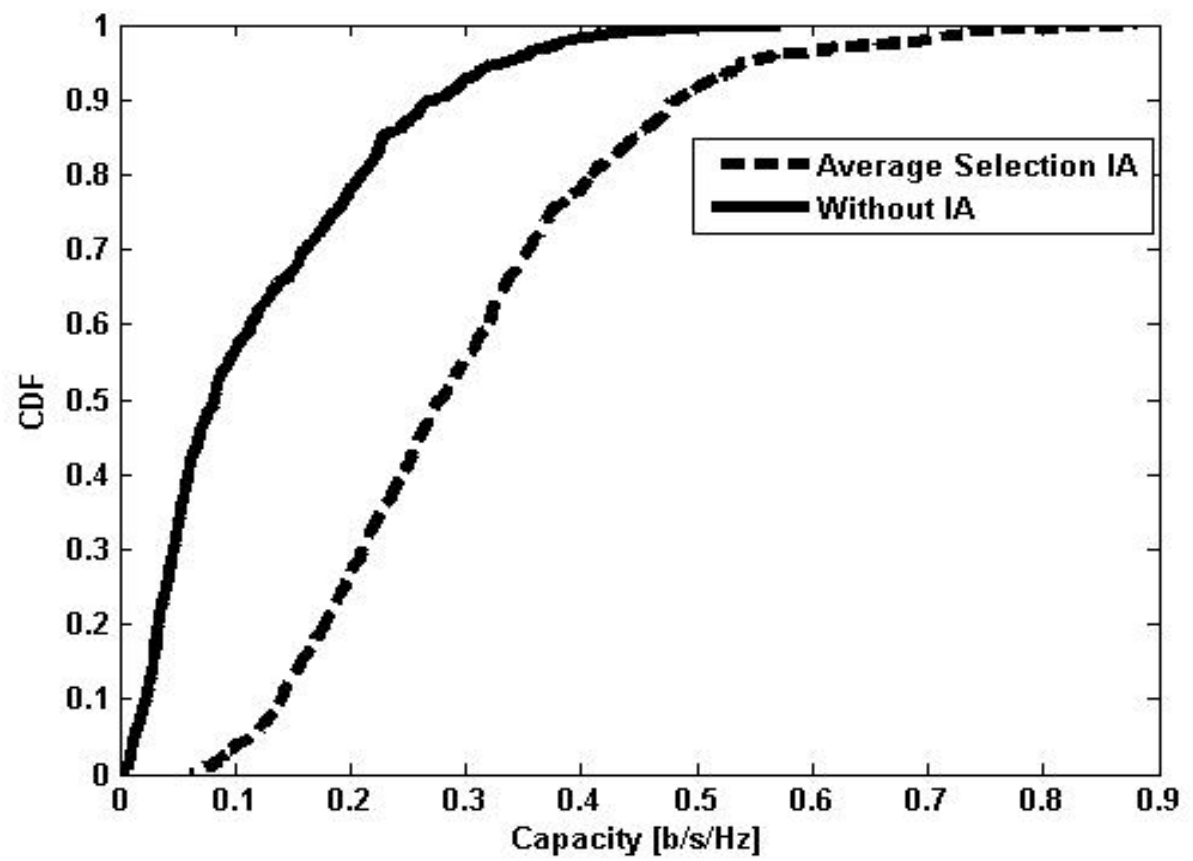

Figure 4-6 CDF of the capacity using the average selection IA 


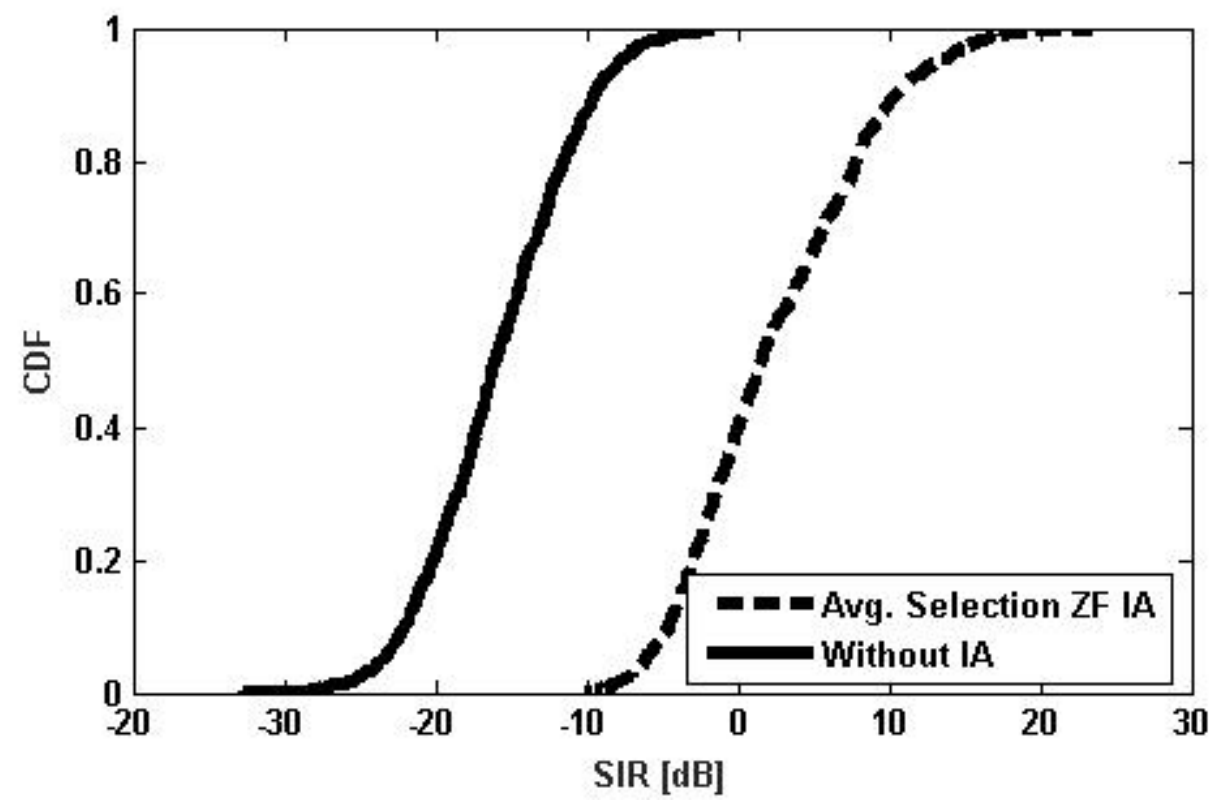

Figure 4-7 CDF of the SIR using the average selection IA and ZF

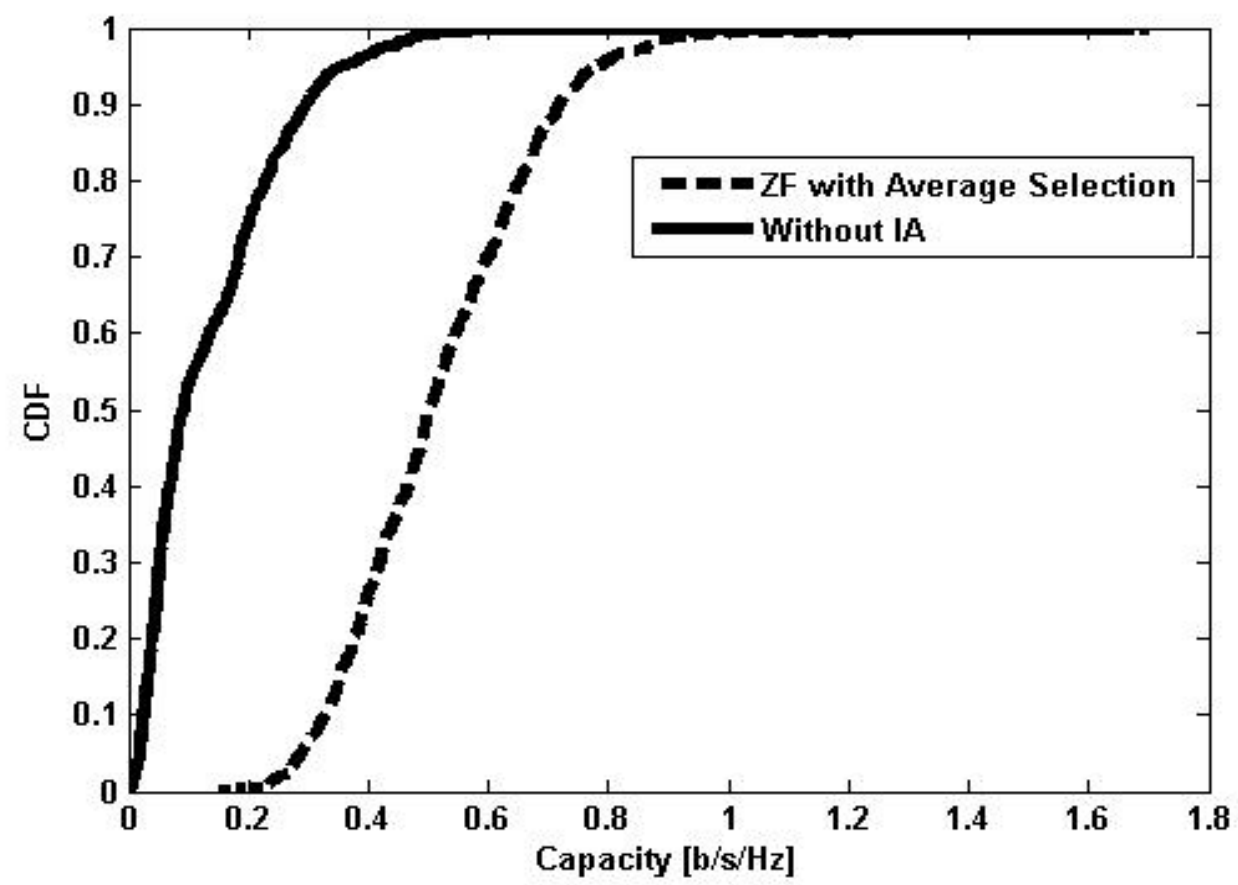

Figure 4-8 CDF of the capacity using the average selection IA and ZF 


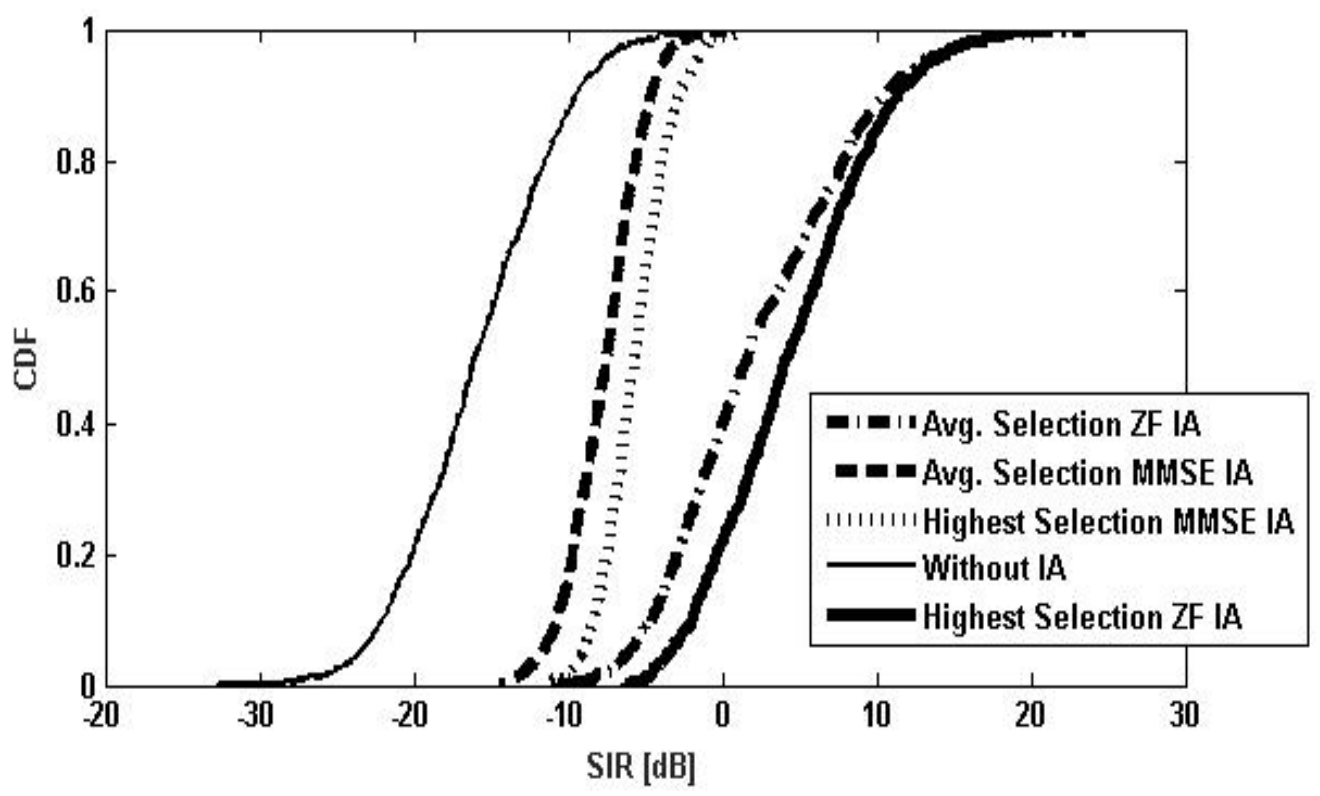

Figure 4-9 CDF of the SIR using the highest selection IA and average selection IA algorithms

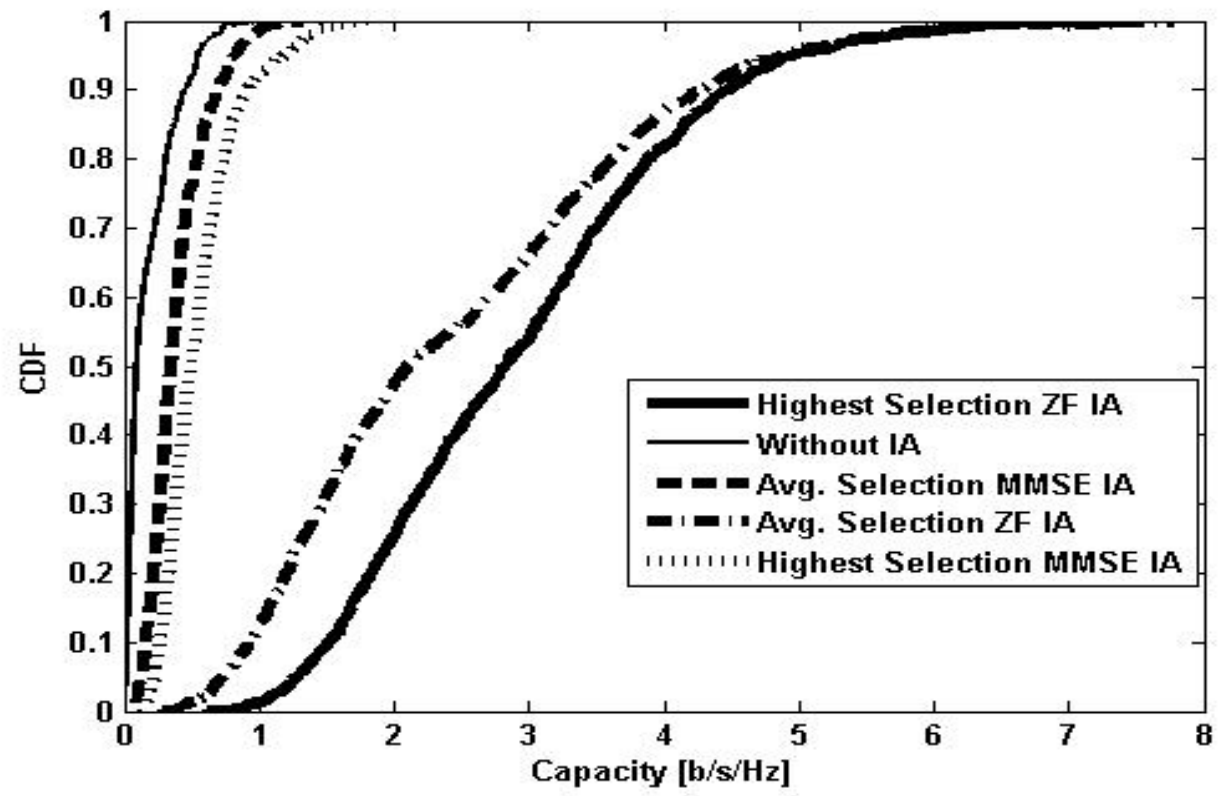

Figure 4-10 CDF of the capacity using the highest selection IA and average selection IA algorithms 


\section{Conclusions and Future Work}

\subsection{Conclusions}

The co-operation of networks with different characteristics and capacities is the main idea of heterogeneous networks which can provide higher data rates, reduced latency and enhanced QoS. However, this networks co-operation results in a multi-tier architecture which suffers mainly from interference.

Femtocells have many advantages as they cover small geographical areas to serve home users with a low cost which reduces the load on the macrocell.

In this paper, a cross-tier uplink interference alignment algorithm has been proposed to reduce the macrocell users interference signals at the femtocells receivers. Since the proposed algorithm align the macrocell users signals whose channels impacts are above a certain threshold, this algorithm is less complex than the one which aligns all the possible interference signals.

Due to the fact that the aligned interference signals are nulled at the receiver using the ZF technique, simulation results show that the proposed algorithm outperforms the other IA algorithms as it significantly increases the SIR and the system capacity.

\subsection{Future Work}

In this paper, the interference due to other femtocells users was treated as noise for simplicity. For the future work, it's recommended to use the proposed average selection IA applying ZF at the FBSs to reduce the cross-tier interference due to macrocell users while taking into consideration the interference due to other femtocells users and trying to mitigate this interference as this will improve the system performance.

It's also recommended to apply the proposed algorithm to reduce the cross-tier downlink interference at the femtocells users. Moreover, it's recommended to evaluate the complexity in a mathematical form.

\section{References}

[1] Bhushan N., Li J., Malladi D., Gilmore R., Brenner D., Damnjanovic A., Sukhavasi R., Patel C., , Geirhofer S., Network densification: The dominant theme for wireless evolution into 5G. IEEE Communications Magazine, 52, 2, 2014, 82-89.

[2] Cao P., Zappone A., Jorswieck E. A.,Grouping-Based Interference Alignment With IACell Assignment in Multi-Cell MIMO MAC Under Limited Feedback, IEEE Transactions on Signal Processing, 64, 3, 2016, 1336-1351.

[3] Chandrasekhar V., Andrews J., Gatherer A., Femtocell networks: a survey, IEEE Commun. Mag., 46, 9, 2008, 59-67.

[4] Chen S., Cheng R. S., Clustering for Interference Alignment in Multiuser Interference Network, IEEE Transactions on Vehicular Technology, 63, 7 2014, 2613-2624. 
[5] Guler B., Yener A., Interference Alignment for Cooperative MIMO Femtocell Networks, Global Telecommunications Conference (GLOBECOM 2011), 2011, 1-5.

[6] Guler B., Yener A., Selective Interference Alignment for MIMO Cognitive Femtocell Networks, IEEE Journal on Selected Areas in Communications, 32, 3, 2014, 439-450.

[7] Guler B., Interference Management For Femtocell Networks, Master of Science thesis, The Pennsylvania State University, 5, 2012.

[8] Hossain E., Rasti M., Tabassum H., Abdelnasser A., Evolution towards 5G multi-tier cellular wireless networks: An interference management perspective, IEEE Wireless Communications, 21, 6, 2014, 118-127.

[9] Li X., Al-Shatri H., Ganesan R. S., Papsdorf D., Klein A., Weber T., Uplink-Downlink Duality of Interference Alignment in Cellular Relay Networks, 10th International ITG Conference on Systems, Communications and Coding, Hamburg, Germany, 2015, 1-6.

[10] Niu Q., Zeng Z., Zhang T., Gao Q., Sun S., Joint interference alignment and power allocation in heterogeneous networks, IEEE 25th Annual International Symposium on Personal, Indoor, and Mobile Radio Communication (PIMRC), Washington DC, 2014, 733-737.

[11] Picochip, The Case For Home Base Stations, White Paper, 4, 2007.

[12] Rezaei F., Tadaion A., Sum-Rate Improvement in Cognitive Radio Through Interference Alignment, IEEE Transactions on Vehicular Technology, 65, 1, 2016, 145154.

[13] Serbetli S., Yener A., Transceiver optimization for multiuser MIMO systems, IEEE Trans. Signal Process., 52, 1, 2004, 214-226.

[14] Talwar S., Choudhury D., Dimou K., Aryafar E., Bangerter B., Stewart K., Enabling technologies and architectures for 5G wireless, Proc. IEEE MTT-S International Microwave Symposium (IMS), 6, 2014, 1-4.

[15] Xia P., Chandrasekhar V., Andrews J. G., Open vs. Closed Access Femtocells in the Uplink, IEEE Transactions on Wireless Communications, 9, 12, 2010, 3798-3809.

[16] Zakrzewska A., Ruepp S., Berger M., Towards converged 5G mobile networks Challenges and current trends. Proc. ITU Kaleidoscope Academic Conference, 6, 2014, 3945.

[17] Zhang J., de la Roche G., Femtocells, Technologies and Deployment, John Wiley \& Sons, 2010. 
[18] Zhao N., Yu F. R., Sun H., Li M., Adaptive Power Allocation Schemes for Spectrum Sharing in Interference-Alignment-Based Cognitive Radio Networks, IEEE Transactions on Vehicular Technology, 65, 5, 2016, 3700-3714.

Received 15.05.2018, Accepted 15.09.2018

This paper is a revised and extended version of work originally presented at the Signal Processing Algorithms, Architectures, Arrangements, and Applications Conference, 21-23 September 2016, Poznan, Poland 